Samuel Fernández E.

Profesor de la Facultad de Teología

Pontificia Universidad Católica de Chile

\title{
Relación del Padre Alberto Hurtado, S.J., con la Facultad de Teología de la Pontificia Universidad Católica de Chile
}

\begin{abstract}
"Es necesario de tiempo en tiempo encerrarse a pensar y repensar para hacer un bien más hondo, más intenso y más extenso".
\end{abstract}

(Carta del 17 de noviembre de 1947).

Al inaugurar el año académico 2002, en que se cumplen cincuenta años de la muerte del Padre Alberto Hurtado, parece oportuno presentar su estrecha vinculación con la Universidad Católica y, en particular, con nuestra Facultad de Teología. Esta vinculación se puede dividir en varias etapas: la primera comprende sus cinco años como estudiante de Leyes; luego, desde Lovaina, su decisiva colaboración para la fundación de la Facultad de Teología; posteriormente, en sus años de ministerio sacerdotal en Chile, su actividad como profesor en Educación, Derecho y Arquitectura, y su abundante predicación de retiros tanto para los profesores como para los estudiantes de la Católica; finalmente, su último testimonio, es decir, su enfermedad y su muerte, se desarrolló en el Hospital Clínico de nuestra Universidad.

Ofrecemos, en primer lugar, los datos históricos, fundamentados rigurosamente en los documentos disponibles, sobre la vinculación de Alberto Hurtado a la UC, su colaboración en la fundación de nuestra Facultad de Teología y, finalmente, su opinión acerca de la formación teológica.

Naturalmente, no se pretende dar una visión general de la rica personalidad del P. Hurtado, sino detenernos en un aspecto específico, como es su relación con nuestra Facultad y su aprecio por la formación teológica.

\section{RELACIÓN DEL P. HURTADO CON LA UNIVERSIDAD CATÓLICA}

\section{A. Período de estudiante de Leyes}

Este período de su vida está marcado por una intensa actividad universitaria, por un decidido apostolado y por un profundo discernimiento vocacional. 
En marzo de 1918, Alberto Hurtado comenzó sus estudios de Leyes en la UC, en ese tiempo contaba con el apoyo espiritual de don Carlos Casanueva, quien desde el año siguiente será rector de la Católica. Durante sus años de estudiante, Alberto se involucró intensamente en la vida universitaria. De sus cinco años de estudio, en cuatro participó en el Centro de Alumnos de Derecho, tal como lo atestigua la Revista Universitaria de aquella época. Ya en esos años Alberto manifiesta su preocupación por los más pobres. El Padre Damián Symon, sscc., su director espiritual, así describe su personalidad cuando era universitario: "Tenía un corazón como un caldero en ebullición que necesita vía de escape" (1).

Por otra parte, los argumentos de sus memorias de grado muestran un interés por abordar académicamente los temas sociales: su tesis de Bachiller trata sobre $\mathrm{La}$ reglamentación del trabajo de los niños, presentada en 1921, y la de Licenciatura, sobre El trabajo a domicilio, presentada en 1923 y publicada ese mismo año por la Revista Universitaria.

Durante estos años realiza un profundo discernimiento vocacional. Testigo de ello son las cartas que Alberto, siendo estudiante, le escribió a su inseparable amigo, Manuel Larraín, compañero de curso en el Colegio San Ignacio y en la Católica, que también será sacerdote, luego Vicerrector de nuestra Universidad y finalmente Obispo de Talca (2).

El 4 de agosto de 1923 aprueba el examen de grado de abogado ante la Corte Suprema, con distinción unánime. Justo antes de entrar al Noviciado jesuita, la Universidad despide a su ex alumno. Los sentimientos de la Universidad están testificados por la Revista Universitaria que nos transmite un documento de inestimable valor, por ser contemporáneo a los hechos, así comienza la crónica:

Después de haber cursado con el más hermoso éxito los cinco años de la Facultad de Leyes, y de haber obtenido brillantemente su título de abogado con nota óptima de la Corte Suprema y distinción unánime de la Universidad Católica, Alberto Hurtado, nuestro amigo, el amigo de todos los jóvenes católicos, el amigo de pobres y ricos, partió al noviciado de la Compañía de Jesús. Su inmenso amor a Dios fue premiado por la Divina Providencia que le concedió el mérito de abandonarlo todo cuando todo podía tenerlo. La Universidad Cató-

(1) "Le conocí cuando ya era universitario [...] Las virtudes que fueron aflorando y solidificándose fueron deslumbradoras, sobre todo la que se refería a la caridad, pues apareció un celo incontenible, que había de moderar repetidamente para que no llegara a la exageración. No podía ver el dolor sin quererlo remediar, ni una necesidad cualquiera sin poner estudio para solucionarla. Vivía en un acto de amor a Dios que se traducía constantemente en algún acto de amor al prójimo, su celo casi desbordado, no era sino su amor que se ponía en marcha. Tenía un corazón como un caldero en ebullición que necesita vía de escape, y aquí está la explicación de esa multiformidad de obras de caridad que las presentía desde joven y que las realizó ya hecho sacerdote y religioso", citado por A. Lavín, El Padre Hurtado, Apóstol de Jesucristo, en Padre Alberto Hurtado, s.j. Tres miradas sobre su vida y su muerte, Santiago 2001, p. 14.

(2) En 1923 Alberto le escribe a su amigo Manuel: "Reza, pero con toda el alma, para que podamos arreglar nuestras cosas y los dos cumplamos este año la voluntad de Dios" (Carta de Alberto Hurtado a Manuel Larraín, s63 y 18 [para citar los manuscritos, se utiliza la nomenclatura del Archivo del P. Hurtado]). Para Alberto, cumplir la voluntad de Dios era entrar al noviciado jesuita, y para Manuel, entrar al Seminario de Santiago. 
lica sintió la necesidad de despedir con todo su cariño al ejemplar ex alumno y celebró en las vísperas de su partida una Misa que ofició el señor Rector y a la cual concurrió un numeroso grupo de sus amigos (3).

Alberto ni siquiera espera el diploma oficial para poder ingresar al Noviciado el día 15 de agosto, elegido por él, por su amor a la Virgen María. Amor que se mantendrá a lo largo de toda su vida.

\section{B. Período de estudiante jesuita}

La primera formación la recibe en Chillán entre ejercicios espirituales y labores humildes; posteriormente comenzará sus estudios en Argentina, para continuar con la filosofía y teología en Sarriá, Barcelona. La tensa situación social obligó a los jesuitas extranjeros a abandonar España. Pasó algunos meses en Irlanda, para mejorar su inglés, y luego continuó sus estudios de teología en la Universidad de Lovaina, una de las más prestigiosas del mundo. Es allí donde se reveló su genio intelectual, graduándose en teología y obteniendo el doctorado en pedagogía con máxima distinción. Según un testigo tan cercano como el Padre Álvaro Lavín: "En Lovaina, y especialmente en sus estudios teológicos, fue cuando comenzó a dar muestras muy claras de una gran capacidad intelectual. Como ya dije, en sus estudios secundarios fue un alumno bueno, pero corriente; en la Universidad sus estudios fueron, sin duda, muy buenos y coronados por el éxito y las buenas notas [...] En cambio, en Lovaina fue muy buen alumno y llamó la atención. Lo digo, porque para mí, que lo conocí y traté tanto, fue una sorpresa desde entonces -y mayor cada día- el verlo de una agilidad mental muy grande y capaz de captar bien las constantes novedades ideológicas y culturales; sorpresa que he considerado siempre solo explicable por una ayuda especial de nuestro Señor" (4).

El 24 de agosto de 1933 fue ordenado sacerdote, y continúa sus estudios de teología y pedagogía. En mayo del año siguiente alcanza el grado de Licenciado en Teología. El P. Janssens, futuro prepósito general de los jesuitas, preside la comisión examinadora. El 10 de octubre de 1935 presenta la Tesis El sistema pedagógico de Dewey ante las exigencias de la doctrina católica, aprobada con gran distinción, obteniendo así el grado de Doctor en Ciencias Pedagógicas en la Universidad de Lovaina. Es en este período cuando presta su decisiva colaboración en la fundación de la facultad de teología. Colaboración que examinaremos más abajo.

\section{Período de apostolado sacerdotal en Chile}

Un vez en Santiago, a inicios de 1936, comenzó su apostolado con los jóvenes y de modo especial entre los universitarios de la Católica (5). Desde su llegada a

(3) Revista Universitaria VIII, 1923, p. 245.

(4) Á. Lavín, El Padre Hurtado, Apóstol de Jesucristo, en Padre Alberto Hurtado, s.j. Tres miradas sobre su vida y su muerte, Santiago 2001, p. 22.

(5) Así se cumplía el sueño del rector Casanueva de ver al Padre Hurtado dedicado -como dice textualmente- "a la gran pesca milagrosa de almas y vocaciones para la Compañía y la Iglesia. 
Chile, el Padre Hurtado comenzó a desarrollar una actividad como profesor en el Colegio San Ignacio, en el Seminario Pontificio y en la Universidad Católica. Fue nombrado profesor de psicología y pedagogía, y daba conferencias públicas sobre psicología del adolescente, conferencias que posteriormente fueron publicadas por la Revista Universitaria. En Pedagogía fue profesor de Filosofía de la Educación, en Derecho, de Cultura religiosa, y en Arquitectura de Sociología. Pero su tarea educativa no se limitó a las clases formales. Una de sus labores decisivas fue la predicación de retiros espirituales. Conservamos varios de los esquemas originales que testifican los múltiples retiros que dio a estudiantes de la Universidad Católica (6). A estos retiros debe sumarse una buena cantidad de conferencias para la comunidad de la UC. En la Semana Universitaria de 1948 habla de la misión del universitario y sobre el sentido del esfuerzo, y en 1949 dicta otra conferencia sobre la psicología del joven de la postguerra (7). A estos datos se podrían añadir otros, pero esta presentación no pretende ser exhaustiva.

\section{Su enfermedad y muerte}

Su último testimonio fue su enfermedad y muerte. También este período lo vivió dentro de nuestra Universidad, en el Hospital de la Católica. Frente a la muerte se revela la profundidad del hombre y frente a la muerte se manifiesta la grandeza de Dios (8). La elocuente muestra de entrega a Dios ante la muerte ha quedado grabada en el Hospital Clínico por medio de los testimonios de los doctores Armas Cruz y Cubillos, médicos que a su vez han formado las siguientes generaciones.

¡Si vieras qué esperanzas tengo cifradas en ti y cómo ansío la hora de verte con nosotros! Hay más campo que nunca”. Carta de Carlos Casanueva a Alberto Hurtado, 13 de diciembre de 1934. La correspondencia relacionada a la fundación de la Facultad de Teología está publicada a continuación del presente artículo.

(6) En 1937 predicó un retiro para alumnos de Leyes; en 1940, uno para los profesores de la UC. El mismo año 1940 dio ejercicios a unos 40 universitarios, y otro a 60. En 1941 predicó a los estudiantes de Leyes y posteriormente a los de Arquitectura. En 1945 estuvo a cargo del retiro preparatorio para la fiesta del Sagrado Corazón, ante el Rector y la comunidad universitaria. En este retiro el P. Hurtado exterioriza su cariño por la Católica, así lo afirma textualmente: "No he creído poder manifestar en mejor forma mi gratitud a esta querida Universidad, que como vosotros yo también puedo llamar mía por haber estudiado cinco años en ella, que repitiendo en esta misma aula las lecciones que aquí aprendí yo hace más de veinte años...". Sin fecha se encuentran las anotaciones de los retiros para estudiantes de Medicina y los de Comercio.

(7) En esta conferencia muestra su cariño por la Universidad y su rector: "Cuando invitado a hablar dudé mucho del tema, pero pensando que venía a la Universidad, vieja casa para mí de tan caros recuerdos, donde iba a encontrar vuestro Rector, que lo fue también mío, maestros que nos son comunes, y una preocupación que más allá de la profesión flota en el ambiente de esta Universidad, un ambiente de grandes ideales, el de La Mayor Gloria de Dios, el servicio de la Iglesia, el servicio de la Patria, que son los motivos que han ido juntando ladrillo a ladrillo lo que es hoy nuestra Universidad".

(8) Cuando supo la noticia de su inminente muerte, según un testimonio contemporáneo, el Padre Hurtado exclamó: “'Cómo no voy a estar contento! ¡Cómo no estar agradecido con Dios! En lugar de una muerte violenta me manda una larga enfermedad para que pueda prepararme; no me da dolores; me da el gusto de ver a tantos amigos, de verlos a todos. Verdaderamente, Dios ha sido para mí un Padre cariñoso, el mejor de los padres". 


\section{COLABORACIÓN DEL P. HURTADO EN LA FUNDACIÓN DE LA FACULTAD DE TEOLOGÍA}

La correspondencia del Padre Hurtado desde Lovaina entrega preciosos datos acerca de sus inquietudes intelectuales y de su contribución a la creación de nuestra Facultad de Teología. En primer lugar, ofrecemos una exposición cronológica de los hechos más relevantes, para luego destacar su significado.

\section{A. Presentación de los datos históricos}

En abril de 1934, el P. Hurtado le escribe al Rector de la UC para manifestarle su deseo de obtener de parte del Gobierno de Chile una comisión oficial para estudiar instituciones educacionales en Europa, antes de regresar a Chile. La comisión fue otorgada ad honorem, y entre octubre de 1935 y enero del 36 el recién doctorado en pedagogía realizó estas visitas con gran dedicación y fruto. De hecho, en agosto de 1938, el P. Hurtado fue llamado a formar parte de una comisión gubernamental del Ministerio de Educación para revisar los contenidos de la enseñanza secundaria. El decreto del nombramiento afirma que "es necesario que el contenido de los programas esté aún más claramente orientado, no a la erudición enciclopédica, sino a la formación de la personalidad del adolescente” (9).

En 1931, había sido publicada por S.S. Pío XI la Constitución Deus Scientiarum Dominus, que proponía una nueva orientación a los estudios de teología. En 1934, don Juan Subercaseaux le escribe al Padre Hurtado para consultarle sobre la conveniencia de adoptar ese nuevo plan de estudios eclesiásticos en el Seminario de Santiago. La respuesta de Alberto Hurtado manifiesta su antipatía al sistema de manuales aprendidos de memoria y su gran aprecio por la investigación científica en teología.

Indudablemente, lo más relevante para nosotros de esta correspondencia es lo que se refiere a la colaboración del Padre Hurtado en la fundación de nuestra Facultad de Teología. Ya desde el nacimiento de la UC, en 1888, se sintió la necesidad de contar con una Facultad de Teología. Durante el rectorado de don Carlos Casanueva se volvió una y otra vez sobre el tema (10). Especialmente después del año 1927 en que fue suprimida la Facultad de Teología de la Universidad de Chile. En la mente del

(9) Decreto del Ministerio de Educación, Dirección General de Educación Secundaria, del 31 de agosto de 1938, firmado por Carlos Atienza. En una carta explica más de este nombramiento: Carta de Alberto Hurtado a Raúl Montes, 11 de septiembre de 1938: "Me han nombrado miembro de una comisión presidida por el Ministro de Educación y formada por Atienza, Alcayaga, Fremel, Peña y Lillo, Darío Salas, García, Néstor Elgueta y un servidor para la reforma del plan de estudios. Nos reunimos semanalmente. Me interesa mucho conocer -por avión- su opinión sobre nuestro programa, sobre la reforma de la enseñanza de las matemáticas, de la física - ¿menos matemáticas?-, de los ramos que habría que recortar. Tengo poca confianza en el éxito: se recortará un poco los programas, pero nada más... pues temen tocar a los sueldos del profesorado, que disminuirían con las horas de clase. Hágame el servicio de mandarme dos números de Estudios del consagrado a la revisión de la enseñanza. Datos, si los tiene sobre la manera de tomar los exámenes en Argentina, Uruguay, Brasil, textos e informaciones que pudiesen apoyar nuestras pretensiones de reforma en el sentido de una mayor libertad y de una formación más humanista”.

(10) Cf. R. Krebs, Historia de la Pontificia Universidad Católica de Chile, Santiago, 1988, vol. I, pp. 373-375. 
Rector Casanueva estaba mejorar los estudios eclesiásticos y "abrir cursos de Religión Superior para seglares, para formar la "elite" católica” (11). En 1934, Alberto Hurtado es consultado acerca de la conveniencia de la fundación de una Facultad de Teología en la Católica, a lo que responde afirmativamente y con mucho entusiasmo. En la UC se había nombrado una comisión especial presidida por el rector e integrada por los presbíteros Eduardo Escudero, Alfredo Silva Santiago, Juan Subercaseaux, Francisco Vives y Manuel Larraín (12). Al mes siguiente, por medio del Vicerrector, Alberto Hurtado recibe el encargo de buscar profesores; para poder comenzar con la Facultad de Teología. Largas y detalladas cartas dan cuenta del enorme esfuerzo realizado por el jesuita chileno para conseguir profesores, a don Carlos Casanueva le escribe desde la cama y le habla de un viaje especial para entrevistarse con profesores que, a pesar de ser un viaje rápido, le exigió 120 horas de tren. La lista de candidatos es larguísima y los resultados no siempre buenos, porque, como destacaremos en seguida, se buscó solo profesores de primer nivel. Llama la atención la certeza de los fundadores de nuestra Facultad en la necesidad de aspirar desde el inicio al carácter universitario de los estudios de teología y al mejor nivel académico.

Junto con el encargo de conseguir profesores, Alberto Hurtado colaboró con la formación de la biblioteca. La falta de medios económicos hacía necesario buscar las grandes colecciones a los precios más convenientes en librerías de libros usados.

La efectiva colaboración del P. Hurtado en la fundación de nuestra Facultad se puede apreciar en las diversas manifestaciones de agradecimiento del entonces Rector de la Católica. El archivo de la rectoría, afortunadamente, ha conservado las cartas del Rector Casanueva al P. Hurtado. En noviembre de 1934 le manifiesta su gratitud:

"Muy agradecido, muy agradecido, y no tengo palabras cómo expresártelo, estamos de tu admirable diligencia para conseguir los profesores de nuestra Facultad de Teología, que tiene ya su casa propia, magnífico palacio a una cuadra de la Universidad y a cuatro del Seminario, instalada ya con todos sus muebles y enseres necesarios" (13).

Asimismo, poco después, en una carta de diciembre del mismo año le declara su agradecimiento en los siguientes términos:

"Con qué pesar me impongo de tu enfermedad, con todas sus consecuencias, pesar tan grande como la inmensa gratitud que te debo por tu empeño tan abnegado, tan inteligente, tan atinado y tan cariñoso, que jamás podré pagarte y solo Dios podrá recompensarte debidamente; después de Dios y de la persona que ha hecho esta fundación (14), a nadie le deberá esta Facultad tanto como a ti y a los Rvdmos. Padres Provinciales de la Compañía, que te han dado tantas facilidades" (15).

(11) Cf. R. Krebs, Historia..., vol. I, pp. 374.

(12) Cf. Actas del Consejo superior, Libro III, sesión del 11 de julio de 1934, citado por R. Krebs, Historia..., vol. I, pp. 375.

(13) Carta de Carlos Casanueva a Alberto Hurtado, 13 de noviembre de 1934.

(14) Se refiere a la señora Isabel Brown Brunet, que donó los \$3.000.000 necesarios para la fundación de la Facultad.

(15) Carta de Carlos Casanueva a Alberto Hurtado, 13 de diciembre de 1934. 
Pero el testimonio más elocuente, e históricamente más seguro, es el discurso oficial del Rector Casanueva en la solemne inauguración de la Facultad, el día $1^{\circ}$ de abril de 1935, discurso publicado en la Revista Universitaria, en el que manifiesta:

"La inmensa gratitud que debía la Universidad al Sacratísimo Corazón de Jesús, al Santo Padre, al Excmo. Sr. Nuncio Apostólico y al Excmo. Sr. Arzobispo, al Rvdmo. Padre General de los Jesuitas, a quien debía mirar nuestra Facultad como a insigne bienechor: al R. P. Alberto Hurtado Cruchaga, que con tanto celo y discreción había cooperado a la elección del profesorado; a los RR. PP. Provincial de los Jesuitas y Salesianos, aquí presentes; el R. P. General de los Agustinos que había dado el ejemplo a todas las Órdenes y congregaciones religiosas en responder a la voluntad de la Santa Sede" (16).

De esta larga lista, el único agradecimiento que va con nombre y apellido es el dirigido al P. Hurtado que ni siquiera estaba presente, porque aún no regresaba a Chile. Este documento contemporáneo a los hechos es una prueba del carácter decisivo de las gestiones realizadas por él. Es importante relevar esta decisiva colaboración en la fundación de la Facultad de Teología, porque no ha sido destacada suficientemente en lo que se ha escrito sobre la historia de nuestra Facultad o acerca de la historia de la Universidad Católica.

\section{B. Visión sistemática}

Con el fin de dar una mirada de conjunto, es posible sistematizar y profundizar los datos anteriormente expuestos en tres apartados:

1. El esfuerzo por lograr el mejor nivel universitario en los estudios de Teología

Este esfuerzo se expresa en primer lugar por el óptimo nivel académico que el Padre Hurtado exige en los posibles profesores de la nueva Facultad de Teología. Una vez recibido el encargo por parte de la Universidad para buscar profesores, se lanzó con gran generosidad en esta empresa. En un primer momento se lamenta de no encontrar doctores "con las condiciones eximias que son de requerir" (17).

Las gestiones las realiza buscando inmediatamente el mejor nivel. Se dirige directamente a los Rectores de las Universidades de Lovaina, Milán, Comillas, de la Gregoriana, del Bíblico, del Seminario de Maynooth, Irlanda. Escribe al P. Jules de Lebreton, a París; se contacta con Strasburgo e Innsbruck, con los Padres Provinciales Jesuitas de Bélgica, Castilla y Toledo; con Padres Dominicos de París; los Padres Franciscanos de Bélgica con muchos profesores de la Universidad de Lovaina y con padres jesuitas de distintos países residentes en Lovaina. Viaja a Valkenburg para ver al Rector de los padres españoles de Aragón, y

(16) Revista Universitaria, XX, mayo-junio 1935, p. 124

(17) Carta de Alberto Hurtado a José Llusá, 15 de agosto de 1934. 
conversar con el Rector del gran teologado alemán, llegando hasta Nimega para ver al Rector de la Facultad de Teología de la Universidad Católica. Estas gestiones resultan verdaderamente agotadoras.

Respecto del nivel de los candidatos es sumamente exigente. Hay que recordar que no todos los centros teológicos habían asumido el desafío de estudiar la teología históricamente y con exigencias universitarias; así se explica la opinión que citamos a continuación:

"Yo he de confesarle -le dice Alberto Hurtado al Rector Casanueva- que en principio tengo alguna desconfianza de los sacerdotes que no tengan más formación que la de Comillas, pues aunque sea excelente para formar buenos sacerdotes y hasta profesores de Seminarios, ella sola me parece insuficiente para profesores de Universidad. Lo mismo diría de la Gregoriana. No creo que se les haya podido dar suficiente entrenamiento en los métodos de trabajo científico, aunque tengan una base ideológica excelente" (18).

Posiblemente, la sensibilidad de Alberto Hurtado por el apostolado intelectual lo impulsaba a considerar insuficiente una formación bien estructurada, como la de Comillas, pero inadecuada para entrar en diálogo con las corrientes de pensamiento ajenas a la Iglesia. Y el ambiente universitario exige hombres capaces de dialogar, con una visión amplia. Pero esta dificultad no descorazona al sacerdote chileno; en otra carta a don Carlos Casanueva le propone una solución:

"Todos estos sacerdotes son antiguos alumnos de Comillas, con muy buena base filosófica y teológica, de talento, jóvenes, celosos, muy buenos sacerdotes que serían excelentes profesores de Seminarios, pero yo creo que les falta una mirada más amplia sobre sus materias, un estudio en Universidades extranjeras, el conocimiento de las lenguas modernas. Con todo esto serían excelentes. De aquí que yo creo que el ideal sería enviarlos un año por cuenta de la Universidad a distintas Universidades extranjeras, v. gr. a Munich o Lovaina al de Historia Eclesiástica, a París al de Fundamental, a Lovaina al de Derecho Canónico, a Roma al de Sagradas Escrituras y entre tanto aceptar profesores suplentes de entre los que le propondré. Si no la formación de la Facultad queda definitivamente coja y sería gran lástima” (19).

La propuesta es audaz: se trata que la pequeña Universidad chilena otorgue becas a profesores europeos ya doctorados, con el fin de ampliarles su formación en vistas de una preparación verdaderamente universitaria.

Entre los invitados a venir a Chile se encuentran grandes maestros como el jesuita francés D'Alés, que en ese tiempo era nada menos que Decano del Institut Catholique de París y Director del Dictionnaire d'Apologétique. Lo sorprendente es

(18) Carta de Alberto Hurtado a Carlos Casanueva, 27 de septiembre de 1934.

(19) Carta de Alberto Hurtado a Carlos Casanueva, 5 de noviembre de 1934. 
que en principio el Padre D’Alés aceptó venir a Chile por algunos meses (20). No conocemos los motivos por los que esta venida no se concretó.

Después de tantas gestiones, los resultados, sin ser óptimos, fueron buenos. Se logró que el profesor de Teología Fundamental de la Gregoriana de Roma se viniera a Chile, llegando a pedir permiso a su superior para quedarse permanentemente en nuestra Facultad. Se trata del Padre Juan María Restrepo, primer Vicedecano y segundo Decano de nuestra Facultad. Asimismo, el Pbr. Dr. don Juan Stelzenberger, profesor de Teología moral de la Universidad Würzburg y el Padre Rodolfo Arbesmann, agustino, profesor de Historia Eclesiástica, griego bíblico y arqueología, vinieron a enseñar a la nueva Facultad. Y así, con la colaboración de un grupo de sacerdotes chilenos, el 1 de abril de 1935, pudo comenzar a funcionar nuestra Facultad (21). Posteriormente, en 1943, en un informe sobre la educación en Chile para el Nuncio, Mons. Zanini, el Padre Hurtado revela su satisfacción en relación a la Facultad de Teología, refiriéndose a ella como la "gloria de la Universidad, y significa un inmenso paso hacia una formación seria del profesorado de seminarios $y$ del clero secular y regular. Gran importancia tiene la asistencia a ella de los estudiantes de varias congregaciones religiosas cuya formación era, anteriormente, muy deficiente" (22).

Otra muestra del aprecio por el trabajo científico fueron las gestiones hechas por el Padre Hurtado para comprar libros para la Biblioteca de la nueva Facultad de Teología (23). Las dificultades económicas eran severas y el Rector Casanueva confiesa su aflicción ante el alto costo de los libros, puesto que, tal como él mismo lo afirma, respecto de los libros, los anhelos del Padre Restrepo no tienen límites (24). A pesar de eso, no se renunciaba a aspirar a una biblioteca de nivel científico.

(20) “El P. D’ Alés, S.J., decano de Teología del Instituto Católico de París, director del Dictionnaire d'Apologétique, autor de seis o siete obras teológicas, aceptaría ir por un año y explicaría el tratado de Verbo Incarnato, o de Deo Trino, o de Eucaristía, no el de Fundamental, que no ha preparado. Yo pienso que sería una gloria para la nueva Facultad el tenerle y que vale la pena a pesar del anticipo de esta materia, que podría darse en lugar de una materia accesoria. El está entusiasmado con la idea de ir, el Padre Provincial no tiene dificultad seria”. Carta de Alberto Hurtado a Carlos Casanueva, 5 de noviembre de 1934.

(21) Cf. A. Martínez, Diez años de la Facultad de Teología, Anales de la Pontificia Facultad de Teología, No 6 (1945), pp. 9-34. La Revista Universitaria de 1935 dedica varias páginas a la Facultad de Teología. También se encuentran datos en C. Casanueva, Memoria de la Universidad Católica de Chile correspondiente a los años 1934-1937, Santiago 1958.

(22) Informe sobre la educación católica en Chile, a Mons. Zanini, 10 de junio, 1943 (s27 y 06).

(23) Cf. Carta de Alberto Hurtado a Juan Subercaseaux, 29 de mayo de 1934: "Se me ocurría proponerle, Mons., si no podríamos hacer algo por entrar en contacto con los libreros para obtener en mejores condiciones los libros y revistas, tanto más cuanto que si se crea la Facultad de Teología será necesario dotarla de ciertas publicaciones modernas. Yo creo que se pueden obtener ventajas considerables. Este junto material, con ser el más desagradable, es, por desgracia tan importante dados los escasos recursos de que disponemos en Chile para estas grandes obras que casi no cuentan más que con la Providencia. Así últimamente he debido entrar en relaciones con la casa editora de la Teología de Van Noort, excelente como libro de texto, pero bastante caro, y me ha prometido un $20 \%$ de rebaja. Para los breviarios obtenemos en casa rebajas de los editores que llegan al $33 \%$ en algunos casos; para las revistas obtenemos muchos intercambios. Este trabajo me parece muy importante, lástima que supone bastante tiempo”.

(24) Cf. Carta de Carlos Casanueva a Alberto Hurtado, 17 de abril de 1935: “En pocos días más te enviaré una segunda remesa en dólares, desde Nueva York, para los libros que pida el Rvd. Padre Juan María Restrepo Jaramillo, que tenemos la honra de tener como profesor de Teología 
Alberto Hurtado aprovecha su estadía en Londres para recorrer librerías de libros usados en busca del Mansi y de colecciones patrísticas tan autorizadas como Die griechischen christlichen Schriftsteller y el Corpus Scriptorum Ecclesiasticorum Latinorum, más conocidos como el Corpus de Berlín y el Corpus de Viena (25). No deja de ser significativo que, en la compra de estas colecciones que hasta hoy utilizamos en nuestra biblioteca y que siguen representando el mejor nivel científico en estudios patrísticos, haya al menos participado el propio Padre Hurtado.

\section{El aprecio por la investigación científica en teología}

En las opiniones de Alberto Hurtado sobre el nuevo plan de estudios propuesto por la Contitución Deus Scientiarum Dominus, se percibe un real aprecio por la investigación científica en teología. La Universidad de Lovaina tenía gran protagonismo en esta renovación teológica que ponía más énfasis al carácter histórico del desarrollo del dogma y reaccionaba, tal como lo dice el Padre Hurtado, "contra el sistema de manuales aprendidos de memoria; y contra el hecho de que la ciencia teológica esté como monopolizada por los protestantes o racionalistas, sobre todo en el terreno de la Sagrada Escritura y Patrística” (26). Esta orientación basada en los manuales la debió "padecer" en Sarriá, Barcelona, donde comenzó sus estudios de teología. Los apunte de clases que se conservan dan cuenta de una teología esquemática y apologética que poco debió entusiasmar al joven jesuita.

Efectivamente, tal como lo afirma Aubert en la historia de la teología de Vorgrimler, "a principios del siglo $X X$, los católicos se hallaban considerablemente rezagados en el campo de la exégesis y de la historia del dogma” (27), y esta orientación más histórica, muy presente en Lovaina, que había estado principalmente en manos protestantes, comenzaba a hacerse un lugar significativo en el ambiente católico. En esos años, Alberto Hurtado tuvo contacto con grandes maestros como D’Alés, Lebreton y Ghellink, que despertaron en él un gran aprecio por la investigación y el trabajo personal en el ámbito de una teología más histórica.

Fundamental en nuestra facultad, procurando acogerte a los precios más bajos que puedan conseguirse por los libreros, ya que no podremos dedicar a este objeto mas de cien mil pesos chilenos para todo, y los anhelos del padre no tienen límites y deseo, en cuanto es de mi parte, satisfacerlos".

(25) Cf. Carta de Carlos Casanueva a Alberto Hurtado, 24 de julio de 1935: "Aquí están muy interesados los profesores de Teología por la obra de Mansi y el "Corpus Patrístico" de Berlín y Viena. ¿A que precios podrían conseguirse estas obras con todos los descuentos y economías del caso? Querría complacerlos, pero me sería imposible si los precios fueran muy cuantiosos; ve en Londres qué obras de Teología podrían obtenerse en los anticuariados". En la carta de respuesta, del 13 de agosto de 1935, Alberto Hurtado afirma "Hoy he pasado el día recorriendo librerías de anticuariado". Más tarde, el Rector insiste: "No te olvides de averiguar los precios más bajos a que pueden obtenerse las ediciones críticas de los Santos Padres de Berlín y Viena de que te hablaba en mi carta anterior. De acuerdo con el Padre Restrepo, limita tus compras a Mansi, para dejar lo más posible para dichas ediciones críticas, que es lo más urgente de todo, pues no existe ni un solo ejemplar en Chile, y no están autorizados los trabajos que no se hagan a base de éstas".

(26) Carta de Alberto Hurtado a Juan Subercaseaux, 17 de abril de 1934.

(27) R. Aubert, en H. Vorgrimler - R. Vander Gucht, La teología en el siglo XX, vol. II (BAC maior 6), Madrid 1973, p. 9. 
3. El deseo de un "Apostolado intelectual” abierto al mundo

El entusiasmo de Alberto Hurtado ante el proyecto de la fundación de una Facultad de Teología en Chile está motivado por su convicción de la necesidad de una muy buena formación teológica para poder evangelizar los ambientes intelectuales de nuestro país. Así se lo expresa al Rector del Seminario de Santiago, don Juan Subercaseaux, en una carta del 17 de abril de 1935:

"Una facultad de Teología en la Universidad, además de completar y coronar los estudios universitarios, nos permitiría tener en Chile un grupito, todo lo reducido que se quiera, pero de mucha competencia en materia de estudios religiosos. A ellos podría acudirse para pedir orientación en los problemas que cada vez más agudos se irán presentando entre nosotros de filosofía religiosa, teología, etc. ¿No sería esta una solución para combinar los estudios fundamentales, tradicionales, sólidos que habrían de darse a la mayor parte de los seminaristas, con los estudios de carácter más profundo, más moderno necesarios para el apostolado intelectual en Chile? Yo creo que entre nosotros hay necesidad de unos cuantos hombres capaces de poder afrontar un apostolado con hombres como los profesores del Pedagógico y Universidad de Chile, con los cuales tenemos tan pocos puntos de contacto por nuestra formación general".

El joven jesuita está convencido de la necesidad de un pequeño grupo de mucha competencia, que haya realizado estudios de carácter profundo y moderno, para poder entrar en contacto con el ambiente univesitario laico, por entonces, tan hostil a la fe y a la Iglesia.

$\mathrm{Su}$ intención de realizar un "apostolado intelectual" se manifiesta también en las gestiones que realiza, ante el entonces Ministerio de Instrucción, para obtener una comisión oficial del Gobierno de Chile para estudiar algún punto útil de los sistemas pedagógicos europeos. Esta mejor formación pedagógica serviría para "acercar al sacerdote al profesorado oficial, acercamiento que ha de ser la primera base de un posible apostolado entre ellos" (28). Con la misma intención le plantea al Rector de la UC la organización de una exposición de libros modernos de pedagogía y la formación de "una biblioteca de pedagogía, donde pudiéramos reunir libros que sirvieran de base aun para las discusiones en pro de la reforma de la enseñanza. Es un punto que tengo muy ante los ojos al buscar libros” (29). Nuevamente se expresa la convicción de la necesidad del mejor nivel académico para entrar en

(28) "Desde hace mucho tiempo vengo pensando en la necesidad, o por lo menos en la gran ventaja que significa para los colegios particulares el ponerse en contacto inmediato con la enseñanza oficial y en primer lugar con el Ministerio de Instrucción. Fuera de las ventajas que esto significa para los colegios particulares trae también consigo la de acercar al sacerdote al profesorado oficial, acercamiento que ha de ser la primera base de un posible apostolado entre ellos. Con este motivo yo desearía obtener una comisión oficial del Gobierno de Chile para estudiar en Europa algún punto útil para la enseñanza nacional, por ejemplo, la Escuela Nueva en algunos países de Europa”, Carta a Carlos Casanueva, 17 de abril de 1934.

(29) Carta de Alberto Hurtado a Carlos Casanueva, 13 de agosto de 1935; cf. Carta a Manuel Larraín, 7 de septiembre de 1934. 
diálogo con los ambientes intelectuales alejados de la Iglesia. Por otra parte, la correspondencia que estudiamos es testigo de la acción mediadora de don Carlos Casanueva entre el P. Hurtado que insiste en la necesidad de comprar libros en inglés y alemán, y el donante de los fondos para la compra, que prefiere invertir en libros en castellano y francés (30).

También busca traer a algunos profesores alemanes "porque podrían ejercer un apostolado intelectual en el terreno no solo de la teología, sino también de la Filosofía”, puesto que los idealistas alemanes tenían mucha influencia en el Pedagógico (31).

Posiblemente, por influencia del espíritu de Lovaina (32), el P. Hurtado con mucha clarividencia comprende que el estudio universitario de la teología no se identifica simplemente con la formación necesaria para el ejercicio pastoral. De ahí, algunas afirmaciones, citadas más arriba, que son fáciles de malinterpretar. Un par de veces afirma que con la sola formación de Comillas se puede ser buen profesor de seminario, pero no de universidad (33). No se trata de infravalorar la enseñanza del Seminario, sino de reconocer lo específico de la labor teológica de nivel universitario, ya que las exigencias del ministerio pastoral no siempre coinciden con las del espíritu universitario. Probablemente, a la base de esta distinción está la inquietud de Alberto Hurtado por el apostolado intelectual, capaz de influir más allá de los límites visibles de la Iglesia.

(30) "Creo que será de todo punto necesario comprar libros en inglés y alemán para la pedagogía, pues en castellano no hay casi nada (da pena); y en francés, es pura vulgarización. En Lovaina todos los autores que vemos son ingleses". Carta a Carlos Casanueva, del 13 de agosto de 1935. En la respuesta se lee: "Respecto a los libros de pedagogía, te incluyo dos cheques que suman $£$ 37.5, que me ha entregado Maximiano Errázuriz para que los inviertas en libros de pedagogía a condición de que queden en la biblioteca del "Hogar de los Estudiantes", fundación que él ha hecho para los alumnos católicos, de pedagogía de Estado, pero no quieren que sean libros alemanes, sino más bien franceses o españoles, porque no poseen ni el inglés ni el alemán en dichos alumno, pudiendo naturalmente usarlos tú como los necesites. No prohíbe, sin embargo, que algunos vengan en inglés; pero como excepción: para los ingleses y alemanes dispón hasta de Fcs. 2.000 belgas, con cargo a los fondos nuestros que tienes en tu poder allá". Carta de Carlos Casanueva a Alberto Hurtado, 23 de agosto de 1935.

(31) "Me parece injustificado un prejuicio general contra todos los alemanes, sobre todo porque podrían ejercer un apostolado intelectual en el terreno no solo de la teología, sino también de la Filosofía, pues los idealistas alemanes han hecho tanto daño en nuestro Pedagógico". Carta a Manuel Larraín, del 7 de septiembre de 1934.

(32) Respondiendo a las consultas de Mons. Subercaseaux sobre la fundación de una Facultad de Teología en Chile, el P. Hurtado le transmite la opinión de uno de sus profesores en Lovaina: “El P. de Ghellink a quien impuse de la carta, me insistía únicamente en un punto, a saber que el número de cursos comunes con el seminario sea el menor posible, para que los estudios de la Facultad sean verdaderamente universitarios". Carta de Alberto Hurtado a Juan Subercaseaux, 29 de mayo de 1934.

(33) Cf. Carta de Alberto Hurtado a Carlos Casanueva, 27 de septiembre de 1934: "Yo he de confesarle que en principio tengo alguna desconfianza de los sacerdotes que no tengan más formación que la de Comillas, pues aunque sea excelente para formar buenos sacerdotes y hasta profesores de Seminarios, ella sola me parece insuficiente para profesores de Universidad". Carta de Alberto Hurtado a Carlos Casanueva, 5 de noviembre de 1934: "Todos estos sacerdotes son antiguos alumnos de Comillas, con muy buena base filosófica y teológica, de talento, jóvenes, celosos, muy buenos sacerdotes que serían excelentes profesores de Seminarios, pero yo creo que les falta una mirada más amplia sobre sus materias, un estudio en Universidades extranjeras, el conocimiento de las lenguas modernas”. 
En cada una de las citas que hemos presentado aparece claro el deseo de realizar un apostolado intelectual en el mundo. No se trata de un estudio que se basta a sí mismo. Se trata de formar una elite intelectual capaz de penetrar los ambientes académicamente más desarrollados del país, representados por la Universidad de Chile y el Pedagógico, que muchas veces eran adversos a la Iglesia.

\section{ALGUNAS OPINIONES DEL P. HURTADO SOBRE EL ESTUDIO Y LA FORMACIÓN TEOLÓGICA}

Pretender una exposición completa de la opinión del P. Hurtado sobre la formación teológica rebasa por mucho los límites de la presente exposición. De todos modos resulta útil ofrecer algunas afirmaciones extraídas de diversos documentos pertenecientes a su época de plena actividad sacerdotal en Chile, incluyendo también algunas que no se refieren directamente a la formación teológica, sino, más ampliamente, al estudio en general.

La acción apostólica del P. Hurtado consistió en su trabajo de formación de personas, especialmente entre los jóvenes, por medio de clases y retiros, y en su labor en el campo social. En ambos ámbitos expresa su aprecio por el estudio.

1. En su trabajo formativo con los jóvenes y adultos, insiste en la importancia del estudio de la propia fe. Organiza inumerables círculos de estudios de la Doctrina Social de la Iglesia, tal como aquellos en que él mismo había participado, en el Colegio San Ignacio, guiado por los padres Vives y Fernández Pradel. Se lamenta del desconocimiento del dogma (34). Y ante un auditorio que quiere escuchar directrices prácticas de corte moral, defiende el carácter práctico del estudio del dogma:

"Hay predicaciones prácticas que consisten en pegar contra todos los vicios actuales (las películas, el escote, el flirteo) y otras que exponen dogmas (y entonces la gente dice: Predicó por las nubes, no fue práctico) pero yo considero que estas últimas son las más prácticas porque cuando conocemos el porqué y la razón de ser de nuestra doctrina, entonces nuestra vida se transforma en función de un ideal" (35).

El mismo apostolado con los jóvenes lo impulsa a él mismo a profundizar sus estudios. En una carta de 1937, dirigida al Padre Raúl Montes, afirma:

(34) Como signo de superficialidad considera "esa falta de vida interior profunda que se respira en tantos hogares... poca o ninguna vida sobrenatural cristiana, piedad superficial, un cristianismo reducido a un grupo de prácticas externas desligadas entres sí y sin sentido profundo... los grandes dogmas desconocidos...!", s53 y 10; cf. s45 y 10; s55 y 17; s57 y 06; s57 y 08.

(35) Retiro del 5 de diciembre de 1943, s47 y 24. Asimismo, en el Mes de María de 1940, hablando sobre la gracia, afirma: "Nuestro público cristiano suele preferir en la predicación que se traten temas aparentemente más concretos, más prácticos como suele decirse: el divorcio, la limitación de nacimientos, una crítica acerba a los escándalos sociales de nuestros días, del casino... etc. Y sin embargo el más práctico de los temas es el de dar a conocer la grandeza de nuestra vida, nuestra auténtica dignidad de cristianos", s48 y 09. 
"Cada día me persuado más que mientras más ahondemos el dogma, la moral y la apologética fundamental, más preparados estaremos para tratar con el prójimo: esto es lo que los jóvenes exigen de nosotros, y hay veces en que uno suda tinta para responderles adecuadamente" (36).

En su invitación a los jóvenes al heroísmo, también el estudio tiene su lugar. Denuncia que "El estudio serio, prolongado, arduo, que exige concentración, es casi imposible en esta vida superficial de continuo paseo" (37). El plan de la Acción Católica contemplaba un estudio sistemático del dogma y la moral cristiana (38). Con vehemencia recuerda a los jóvenes su deber de estudiar:

“¡Cumplir con el deber! El soldado muere en el frente. Moscardó dejó matar a su hijo. El médico, contagiado. El sacerdote, agotado... ¡Ese es el deber! El tuyo: ¡estudiar! ¡La tierra no alimenta inútiles! Todos a trabajar. ¡Comerás el pan con el sudor de tu frente! ;La lección no me gusta! Pero cavar no agrada al labrador, ni pintar al pintor, ni trabajar en verano... ni aplicar los rayos X... ¡y lo hacen! Y solo así se surge" (39).

En medio de exigencias desbordantes recuerda que "Los llamados al apostolado necesitan consagrar sus mejores años a la labor oculta de oración, meditación y estudio" (40), y enumera entre los pecados del hombre de acción "Abandonar el estudio, abandonar la oración, perder la humildad” (41).

En un documento dirigido a seminaristas, en que afirma "Es más de temer en un sacerdote la ignorancia que el pecado"; insiste en el carácter espiritual del estudio: Recuerda que "El espíritu de la Sagrada Escritura solo se abre al estudio reflexivo, constante y lleno de cariño [...]. Debe ser a un tiempo estudio y oración, reflexión e investigación, si ha de abrir el contenido del cristianismo". El estudio serio debe ser también oración, puesto que "Un concepto puramente intelectualista y racional empobrecen la teología”, y declara, citando las palabras del P. de la Colombiere: "Si volviera a la Teología, la estudiaría más de rodillas” (42).

2. Ante el problema social tan urgente y por el cual tenía una sensibilidad tan aguda, también revela su valoración del estudio. En primer lugar busca, tanto en la Escritura como en la Tradición y el Magisterio, las raíces dogmáticas de la acción de la Iglesia, y recuerda que "el problema social es un problema de origen dogmáti-

(36) s62 y 063

(37) A. Hurtado, Humanismo Social, Santiago 1994, p. 135.

(38) Cf. s19 y 05.

(39) s39 y 02d. También s54 y 18: "Vencerse, formarse y formar. Estudiar con sacrificio. No ser del montón... Es tan triste ver que jóvenes renuncian a ser algo, renuncian a influir por falta de cultura. ¡Formarse, leer!”.

(40) s56 y 11.

(41) s45 y 27.

(42) Las citas de todo el párrafo pertenecen al s40 y 11. El documento se titula La formación del sacerdote, no tiene fecha ni contexto, pero al carecer de referencias a los votos religiosos, está posiblemente dirigido a Seminaristas diocesanos. 
co” (43). Por otra parte, insiste en la necesidad de estudiar antes de actuar. Como buen hijo de san Ignacio, recuarda la necesidad de prepararse ante cualquier empresa grande, y para ello propone como ejemplo la vida oculta de Jesús. Una breve y contundente cita basta para mostrar el valor que el P. Hurtado da al estudio, incluso ante una realidad que llama a la acción:

"¿Qué hacer ante el problema social? Hemos visto el pavoroso problema. Soluciones: Ante nada resolverse a estudiarlo" (44).

Y luego recuerda las palabras del Papa Pío XI y las recomendaciones de los obispos sobre el deber gravísimo, en la hora actual, del estudio del problema social. Una actitud semejante se expresa en una carta desde París, desde la residencia Études, donde se encierra durante dos meses para estudiar todo lo que ha recibido en una serie de Congresos y Semanas de Estudio sobre temas sociales en que ha participado a fines de 1947:

"Le escribo a las 5 en un día frío y ya obscuro y pienso con cierta nostalgia en esos hermosos días de Noviembre, llenos de luz y sol, días de mes de María de Santiago... Pero es necesario de tiempo en tiempo encerrarse a pensar y repensar para hacer un bien más hondo, más intenso y más extenso" (45).

Efectivamente, para hacer un bien más hondo, más intenso y más extenso es necesario encerrarse a pensar y repensar (46). En otra ocasión, oponiéndose a la fundación de otro colegio, le insiste al Padre General de los jesuitas en la necesidad de contar con un residencia para los estudiosos: "nos falta tanto una casa de estudios para un trabajo más científico y para una influencia seria” (47). Una vez más, se demuestra que el P. Hurtado estaba convencido que la influencia seria se logra por medio del estudio científico.

\section{CONCLUSIÓN}

La más inmediata conclusión es reconocer la labor decisiva del Padre Hurtado en la fundación de nuestra Facultad de Teología. Labor que no ha sido destacada suficientemente y a veces ni siquiera mencionada en lo que se ha escrito sobre la historia de nuestra Facultad (48).

(43) s24 y 04.

(44) s24 y 03.

(45) Carta del 17 de noviembre de 1947 (s65 y 15).

(46) En una carta del 9 de diciembre de 1947 afirma: "Aquí me tiene en París haciendo vida de Casa de Ejercicios, encerrado en una pieza, lleno de libros, con decirle que ayer no fui a ver el entierro del General Leclereq, que fue algo grandioso: todo París, comenzando por el Presidente, Cámaras, Cardenal... Era hombre de comunión diaria, padre de familia numerosa y ejemplar. Pero hay tanto que hacer, tanto que leer y meditar, pues este viaje me lo ha dado Dios para que me renueve y me prepare en los tremendos problemas que por allá tenemos" (s65 y 16).

(47) s62 y 14. Se trata de una carta del P. Hurtado al P. J.B. Janssens, del 27 de junio de 1950.

(48) En los estudios acerca de la historia de la Facultad de Teología, casi no se menciona al P. Hurtado. Cf. J. Jiménez, Apuntes para la historia de nuestra Facultad de Teología, T y V XVI 
Por otra parte, al detenernos en este punto específico de la rica y variada personalidad del Padre Hurtado, se nos manifiesta una faceta suya, para muchos desconocida. Los documentos estudiados nos muestran un hombre con gran interés y aprecio por el estudio serio y por la investigación; convencido de que para entrar en diálogo con la sociedad actual y colaborar en las solución de sus graves y urgentes problemas, no basta un puñado de fórmulas aprendidas de memoria o un simple entusiasmo afectivo, sino que se requiere una profunda y amplia comprensión del mundo y de la revelación cristiana. Este legado nos ayuda a perfilar más nuestra identidad de Facultad en lo específicamente teológico, tal como lo afirmaba nuestro Decano, el prof. Juan Noemi, en la cuenta anual correspondiente al año 2000 (49). La gran obra externa y visible del P. Hurtado, realizada en pocos años, no es fruto de la improvisación entusiata. Sus acciones estuvieron respaldadas por una profunda vida de oración, un intenso amor por los más pobres y también por el estudio serio.

Por ultimo, resulta extremadamente significativo que, precisamente en nuestra Iglesia chilena, Iglesia marcada por una gran sensibilidad por las urgencias que la rodean y por ello acechada por la tentación del inmediatismo en la acción, uno de los sacerdotes cuyo ministerio pastoral ha sido más fecundo, según nuestros pobres criterios de juicio, le haya dedicado tanto tiempo y con tanta seriedad a su formación teológica y, más en general, al estudio. Al parecer, la formación seria resulta fecunda para la pastoral.

Ciertamente, la fecundidad del ministerio sacerdotal del P. Hurtado no depende solo del estudio, sino principalmente de su santidad de vida, pero no es menos cierto que el Dios providente ha querido suscitar en nuestra Iglesia como ejemplo de santidad (y ha coronado su acción con grandes frutos) a un sacerdote que enfrentó con gran seriedad su formación teológica y manifestó tanto aprecio por el estudio durante su arduo ministerio sacerdotal. De este modo se manifesta como insostenible la tan frecuente oposición entre el estudio serio y el trabajo pastoral.

(1975) pp. 221-240; 316-335; A. Martínez, Diez años de la Facultad de Teología, Anales de la Facultad Pontificia de Teología, nº 6 (1945), pp. 9-34; D. Iglesias, Cinco lustros, Anales de la Facultad de Teología, no 12, pp. 102-110; M. Barrios, La Facultad de Teología de la Pontificia Universidad Católica de Chile. Sesenta años de historia al servicio de Chile y de su Iglesia (1935-1995), Santiago 1995. Este último estudio publica en apéndice parte de la correspondencia del P. Hurtado relativa a la fundación de la Facultad.

(49) J. Noemi, Memoria del año académico 2001, Epílogo, 26 de marzo de 2002. 